\title{
Manifestações Articulares em Pacientes com Doença de Crohn e Retocolite Ulcerativa
}

\section{Articular Manifestations in Patients with Crohn's Disease and Ulcerative Colitis}

\author{
Cristina Costa Duarte Lanna ${ }^{(1)}$, Maria de Lourdes Abreu Ferrari(2), Marco Antônio Parreiras de Carvalho ${ }^{(3)}$, \\ Aloísio Sales da Cunha ${ }^{(4)}$
}

\section{RESUMO}

Os sintomas articulares constituem a manifestação extra-intestinal mais comum em pacientes com doença de Crohn e com retocolite ulcerativa. Reconhecida como artrite colítica, e mais recentemente, artrite enteropática, está classificada como uma das doenças do grupo das espondiloartropatias. Reconhecidamente, há dois padrões de acometimento articular: 1) periférico, habitualmente, associado aos períodos de atividade da doença intestinal e sem associação com o antígeno HLA B27, e 2) axial, caracterizado por espondilite e sacroiliíte, com curso clínico e radiográfico independente da doença intestinal e associado ao HLA B27.

Palavras-chave: artrite, doença inflamatória intestinal, espondiloartropatia, doença de Crohn, retocolite ulcerativa.

\section{INTRODUÇÃO}

Na sua conceituação mais ampla, a doença inflamatória intestinal (DII) corresponde a qualquer processo inflamatório envolvendo o trato gastrintestinal, seja ele agudo ou crônico. A doença de Crohn (DC) e a retocolite ulcerativa (RCU) representam as duas principais formas de apresentação da DII, de causa desconhecida ${ }^{(1)}$. A despeito de constituírem doenças distintas, compartilham aspectos clínicos e epidemiológicos, sugerindo a possibilidade de fatores etiológicos comuns. O curso clínico, cujas manifestações principais são diarréia, dor abdominal e sangramento retal, caracteriza-se por períodos de remissão e exacerbação, e apresenta complicações as mais diversas. Associam-se, com certa freqüência, manifestações extra-intestinais tais como articulares, cutâneas, oculares, hepatobiliares e vasculares, que podem preceder, acompanhar ou suceder a doença intestinal. A etiopatogenia da DII é multifatorial, com a

\begin{abstract}
Joint involvement is the most commom extraintestinal manifestation in patients with Crohn's disease and ulcerative colitis. The colitic arthritis, lately called enteropathic arthritis, is classified as one of the diseases of the spondyloarthropathies group. There are two patterns of joint involvement: 1) peripheral arthritis, usually coincides with exacerbations of the inflammatory bowel disease and is not associated with the HLA B27 antigen; and 2) axial involvement, characterized by spondylitis and sacroiliitis, which the clinical course is independent of the intestinal disease, and has an association with HLA B27.
\end{abstract}

Keywords: arthritis, inflammatory bowel disease, spondyloarthropathy, Crohn's disease, ulcerative colitis.

participação de fatores genéticos, intraluminais, alterações na barreira do epitélio intestinal e resposta imunológica anormal da mucosa. Estes fenômenos determinam a ativação da cascata imunoinflamatória, que resulta em lesão continuada da mucosa do intestino ${ }^{(2,3)}$.

Os sintomas articulares constituem a manifestação extra-intestinal mais comum em pacientes com RCU e DC. Apesar do conhecimento da existência de uma poliartrite em associação às duas enfermidades por décadas, até 1959 , acreditava-se que isto refletia a co-existência delas com a artrite reumatóide $(\mathrm{AR})^{(4)}$. Nesta época, o quadro ficou mais bem definido, quando Wright e Watkinson ${ }^{(5)}$, estudando 108 pacientes com RCU, identificaram em 15,7\% deles um tipo de artrite com características diferentes da AR. Denominaram-na artrite colítica e assim a descreveram: sinovite aguda, recorrente, usualmente monoarticular e assimétrica, de curta duração, que não produz deformidade ou destruição articular.

\footnotetext{
Serviços de Gastroenterologia (Instituto Alfa de Gastroenterologia) e de Reumatologia do Hospital das Clínicas da Universidade Federal de Minas Gerais (UFMG). Recebido em 07/04/05. Aprovado, após revisão, em 06/01/06.

1. Reumatologista, Professora Adjunta do Departamento do Aparelho Locomotor da Faculdade de Medicina da Universidade Federal de Minas Gerais

2. Gastroenterologista, Professora Auxiliar de Clínica Médica do Departamento de Clínica Médica da Faculdade de Medicina da UFMG.

3. Reumatologista, Professor Adjunto do Departamento do Aparelho Locomotor da Faculdade de Medicina da UFMG.

4. Gastroenterologista, Professor Titular de Clinica Médica do Departamento de Clínica Médica da Faculdade de Medicina da UFMG.

Endereço para correspondência: Cristina Costa Duarte Lanna, Rua Juiz da Costa Val, 65/402, Bairro Santa Efigênia, Belo Horizonte, CEP $30240-350$, MG, Brasil, telefone: (31) 3281-8190, fax (31) 3221-1552, e-mail: lanna@medicina.ufmg.br
} 
Em 1964, a então Associação Americana de Reumatismo (ARA) considerou a artrite associada à DC e à RCU um quadro distinto da AR, baseando-se em manifestações clínicas, laboratoriais, radiográficas, e na consistente ausência do fator reumatóide no soro dos pacientes ${ }^{(6)}$. Em 1973 surgiram os primeiros relatos da associação entre o HLA B27 e a espondilite anquilosante (EA), seguindo-se a identificação desta associação com as outras doenças classificadas como espondiloartropatias ${ }^{(7-9)}$. A força da associação com o HLA B27 difere entre as doenças classificadas como espondiloartropatias e sua presença se correlaciona com determinadas manifestações clínicas, como, por exemplo, o envolvimento do esqueleto axial ${ }^{(10-12)}$. Somente em 1974, Moll et al ${ }^{(13)}$ propuseram, com base em critérios clínicos e genéticos, a introdução das artrites enteropáticas no grupo das espondiloartropatias, enfermidades que compartilham similaridades clínicas, tais como: alta incidência de sacroiliíte, predisposição genética e ausência do fator reumatóide ${ }^{(14-17)}$.

Ao consultar a literatura, observa-se grande variação de $2,8 \%$ a $62 \%$ - na freqüência das manifestações articulares associadas à DC e à RCU, predominando os índices de 14,3\% a $44 \%^{(2,5,6,14,18-32)}$. A interpretação dos resultados dos estudos que analisam a freqüência das manifestações articulares em indivíduos com DC e RCU deve ser criteriosa. É necessário levar em conta as diferenças relativas à seleção dos pacientes, ao desenho da investigação, à técnica radiográfica empregada e ao tempo de evolução da doença intestinal. Vários estudos são retrospectivos e diversos trabalhos foram realizados antes da definição dos critérios descritos pelo Grupo Europeu de Estudos em Espondiloartropatia ${ }^{(33)}$, para o diagnóstico e classificação das espondiloartropatias.

Do nosso conhecimento, há três estudos no Brasil sobre o tema. Dois são trabalhos retrospectivos, com apresentação das freqüências das manifestações articulares em pacientes com RCU, realizados por Goldfarb et $\mathrm{al}^{(34)}$ de 1985 , e em pacientes com DC e RCU de Souza et al ${ }^{(35)}$, de 2002. O outro, em um estudo transversal de 130 pacientes com DC e RCU, - descreve manifestações articulares, cutâneas e oculares, além da pesquisa do HLA B27(36).

A maioria dos pacientes com espondiloartropatia não apresenta sintomas ou sinais de inflamação intestinal. Lesões inflamatórias no intestino, no entanto, têm sido identificadas pela histologia em $60 \%$ dos pacientes com espondiloartropatia, mesmo na ausência de qualquer sinal ou sintoma intestinal. As lesões endoscópicas mais raras e constituídas, principalmente, de pequenas erosões, encontram-se presentes em apenas $30 \%$ dos pacientes ${ }^{(14,37-40)}$.

Dois tipos de inflamação têm sido descritos: um tipo agudo, que lembra uma colite infecciosa, e outro com características crônicas, que se assemelha à inflamação da DC. A forma aguda é caracterizada por preservação da arquitetura da mucosa e infiltrado do epitélio composto por neutrófilos e eosinófilos. Na lâmina própria, há edema e infiltrado de polimorfonucleares. Na forma crônica, as criptas estão distorcidas, há atrofia na superfície das vilosidades e o infiltrado da lâmina própria tem população celular mista. O tipo agudo tem sido identificado nos pacientes com artrite reativa secundária a uma infecção intestinal bacteriana e as lesões com características crônicas são mais prevalentes nos pacientes com EA ou com espondiloartropatia indiferenciada ${ }^{(38,40)}$.

A semelhança entre o padrão de acometimento articular dos pacientes com DC e RCU com o de indivíduos com espondiloartropatia, que apresentam envolvimento subclínico do intestino, sugere a possibilidade de a inflamação intestinal ter um papel patogenético na artrite. O reconhecimento do elo imunológico entre espondiloartropatia e DII promoveu o desenvolvimento de novas terapêuticas para as espondiloartropatias ${ }^{(41)}$.

\section{MANIFESTAÇÕES ARTICULARES NA DOENÇA DE CROHN E RETOCOLITE ULCERATIVA}

São reconhecidos dois padrões principais de acometimento articular na DC e na RCU. O primeiro seria uma forma de artrite periférica chamada artrite enteropática. $\mathrm{O}$ segundo padrão, denominado axial, seria a sacroiliíte com ou sem espondilite ${ }^{(24,42-44)}$. Além disso, podem ocorrer manifestações periarticulares como entesite, tendinite e periostite.

Parece claro que a artrite periférica e o acometimento axial em pacientes com DC e RCU são formas clínicas distintas. Enquanto o envolvimento axial pode preceder o início da doença intestinal em anos, isso é raro na forma de artrite periférica. $\mathrm{O}$ curso da espondilopatia não está relacionado à atividade da doença intestinal, enquanto episódios de artrite periférica refletem períodos de atividade inflamatória da doença intestinal ${ }^{(4,44)}$.

\section{ARTRITE PERIFÉRICA ASSOCIADA À DOENÇA DE CROHN E À RETOCOLITE ULCERATIVA}

A artrite periférica em pacientes com DC e com RCU ocorre em uma freqüência que varia de $2,8 \%$ a $31 \%$ nos diversos trabalhos publicados ${ }^{(5,20-26,28-30,32,34,45)}$. A histologia da membrana sinovial revela, em geral, uma reação inflamatória 
inespecífica, incluindo hipertrofia de vilosidades, edema e infiltrado linfohistiocitário ${ }^{(46)}$. Outros autores descreveram a existência de sinovite granulomatosa ${ }^{(47,48)}$.

Em geral, o curso clínico da artrite periférica é autolimitado, não evoluindo com destruição articular, e tende a ser recorrente de acordo com os períodos de exacerbação da atividade clínica da doença intestinal ${ }^{(2,22,24,26,42,43)}$. As articulações mais comumente afetadas no tipo de artrite sem espondilite são os joelhos e os tornozelos $(18,20-22,24,26,28-30,42)$.

Em 1998, foi sugerido por Orchard et $a^{(26)}$ que a artrite periférica poderia ser subdividida em três padrões: tipo I, oligoartrite assimétrica; tipo II, poliartrite simétrica e tipo III, associada à espondiloartropatia. A artrite do tipo I afeta menos de cinco articulações, tem caráter agudo e autolimitado, dura menos que dez semanas e acompanha a atividade inflamatória do intestino. A artrite do tipo II acomete cinco ou mais articulações, os sintomas podem durar meses ou anos, não reflete a atividade da doença intestinal e raramente precede o seu diagnóstico. De acordo com $\operatorname{Khan}^{(16)}$, cerca de um quarto dos pacientes com artrite periférica apresentam envolvimento axial associado.

A artrite periférica pode ser crônica e erosiva em 10\% dos pacientes $^{(49)}$. Maher et a ${ }^{50)}$ publicaram o caso de um indivíduo com RCU que desenvolveu subluxação das articulações metacarpofalangianas e metatarsofalangianas, sem erosões ósseas. Norton et $a^{(51)}$ descreveram três pacientes com artropatia atípica, que apresentavam erosões, destruição e deformidades articulares em associação com a DC. Além destes, Boyer et al ${ }^{52)}$ relataram o caso de uma mulher com RCU e artrite simétrica de pequenas articulações das mãos e pés, com erosão, que preenchia critérios para o diagnóstico de AR. No Brasil, em trabalho desenvolvido por Lanna ${ }^{(36)}$, uma paciente de 46 anos de idade, com doença de Crohn há 23 anos, apresentava uma forma de poliartrite crônica, simétrica, de grandes e pequenas articulações, com 20 anos de evolução, e tinha diminuição do espaço articular em articulações dos carpos, joelhos e tarsos. Havia, ainda, sacroiliíte grau II bilateral e o fator reumatóide e o HLA B27 eram negativos.

\section{ENVOLVIMENTO AXIAL ASSOCIADO À DOENÇA DE CROHN E À RETOCOLITE ULCERATIVA}

O envolvimento axial inclui a espondilite e a sacroiliíte, e é, geralmente, mais comum em pacientes com DC (5\% a $22 \%)$ do que com RCU $(2 \% \text { a } 6 \%)^{(4)}$. Em contraste com os sintomas da artrite periférica, a sintomatologia e o curso clínico da sacroiliíte e da EA são independentes da evolu- ção da doença intestinal e apresentam um curso crônico e

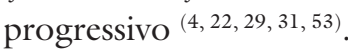

De maneira geral, a EA ocorre em freqüência de 3\% a $15 \%$ dos doentes com DC ou RCU(23,24,28,29,31).

Os sintomas da EA associada à DC e à RCU são similares àqueles vistos na EA idiopática. A faixa etária de início dos sintomas da forma idiopática é abaixo dos 40 anos e, nos casos associados à doença inflamatória intestinal, pode ocorrer em qualquer idade ${ }^{(4,23)}$. A espondilite idiopática é mais comum em homens (2-4:1). Naquela associada à DC e à RCU, entretanto, a relação entre os sexos é $1: 1^{(44)}$. Radiograficamente, a EA associada à DC e à RCU não pode ser diferenciada dos achados da forma idiopática da doença. Além disso, a EA da DC e da RCU tem associação com o HLA B27, o que não ocorre com a artrite periférica ${ }^{(4,29,44)}$. Raramente a EA ocorre na ausência de artrite ou sacroiliíte.

As articulações sacroilíacas são afetadas de forma uni ou bilateral, com intensidade variando desde inflamação leve até anquilose, em freqüência que varia de $6 \%$ a $43 \%$ nos diversos trabalhos publicados, principalmente em associação com a $\operatorname{artrite~periférica~}^{(6,19,21,22,25,28,29,31,32,40)}$. A variação deve-se a diferenças metodológicas entre os estudos. A evidência radiográfica de sacroiliíte em pacientes com DC e RCU é mais comum do que o envolvimento da coluna e pode ser assintomático $^{(6,20-22,25,29,54,55)}$. O tipo do acometimento das articulações sacroilíacas difere entre as enfermidades do grupo das espondiloartropatias. É bilateral e simétrico na EA e nas doenças inflamatórias intestinais, e unilateral ou bilateral assimétrico na artrite reativa, na artrite psoriásica e na espondiloartropatia indiferenciada ${ }^{(15)}$. Somente na EA há forte associação com o HLA B27 $7^{(22,25,29,40,55,56)}$.

Consultando a literatura nacional e da América Latina identificamos poucos artigos que descrevem as manifestações articulares nos pacientes com DC e RCU. Em 1985, Goldfarb et al ${ }^{34)}$ descreveram a experiência de 30 anos do Hospital dos Servidores do Estado do Rio de Janeiro. De forma retrospectiva, foram identificados 98 pacientes com RCU, 14 dos quais $(14,3 \%)$ apresentavam manifestações articulares atribuíveis à doença intestinal. Treze pacientes eram do sexo feminino, 12 eram brancos e dois eram pardos. Em 10 pacientes houve artrite e nos outros quatro apenas artralgia. Em 64,3\% deles a artrite aparecia em crises agudas, regredindo após 10 dias. $\mathrm{O}$ acometimento articular foi simétrico em $60 \%$, comprometendo os joelhos, as mãos, os punhos, os cotovelos, os ombros, os tornozelos, a coluna cervical, os pés, as articulações temporomandibulares, as articulações sacroilíacas e as coxofemorais em ordem decrescente de prevalência. O padrão da artrite foi caracterizado como poliarticular. 
O trabalho de Souza et al, de $2002^{(35)}$, teve o objetivo de analisar a freqüência da admissão de casos da DC e da RCU em hospital de Ribeirão Preto, num período de 20 anos, além de descrever as características clínicas e demográficas destes. Os autores analisaram 126 pacientes com DC e 131 com RCU, e identificaram manifestações articulares em 37 deles ao todo $(14,4 \%)$.

O estudo desenvolvido por Lanna ${ }^{(36)}$, no Hospital das Clínicas da UFMG, analisou 130 pacientes com doença inflamatória intestinal - DC ( $\mathrm{n}=71)$ e RCU $(\mathrm{n}=59)$-, atendidos de forma consecutiva, no período de agosto de 2000 a março de 2003. Identificou freqüência elevada de manifestações articulares - 41,5\% no total. Entre os 130 indivíduos, artrite periférica ocorreu em 25,4\% (29,6\% com DC e 20,3\% com RCU) e acometimento axial em $15,6 \%$ ( $19,7 \%$ com DC e $8,5 \%$ com RCU). As articulações periféricas mais acometidas foram joelhos $(56,1 \%)$, tornozelos $(29,3 \%)$ e coxofemorais $(29,3 \%)$. Predominou o padrão do acometimento articular assimétrico $(65,6 \%)$ e oligoarticular $(84,6 \%)$. Dor lombar inflamatória ocorreu em 13 (10\%) pacientes no total: 8 (11,3\%) com DC e $5(8,5 \%)$ com RCU. Oito dos 13 pacientes preenchiam critérios para a classificação de EA, todos com DC, sendo 6 do sexo masculino. Alterações radiográficas das articulações sacroilíacas foram identificadas em $12 / 130$ pacientes $(9,2 \%)$; sendo em 10 (14,1\%) pacientes com DC e em $2(3,4 \%)$ com RCU. Na maioria deles, os achados eram bilaterais $(91,7 \%)$.

\section{CORRELAÇÃO ENTRE LOCAL DO INTESTINO ACOMETIDO E PRESENÇA DE ARTRITE}

Alguns autores $(20,24,28,57)$ observaram maior incidência de artrite nos pacientes com DC de localização colônica do que naqueles com acometimento isolado do intestino delgado. Entretanto, Münch et al ${ }^{(2)}$, ao estudarem 167 pacientes com DC, não encontraram predominância de uma forma particular de acometimento articular em correlação com o segmento intestinal inflamado.

Em relação aos pacientes com RCU, Wright e Watkinson $^{(18)}$, ao examinarem 209 pacientes, identificaram que havia associação entre a artrite e a forma difusa de acometimento do cólon (pancolite) em pacientes do sexo feminino. Mais recentemente, Scarpa et $a^{(6)}$ ao estudarem 79 pacientes com a forma ativa da doença e artrite, observaram que o número de articulações afetadas estava correlacionado com a forma de acometimento intestinal. Os doentes com proctite tinham mais articulações envolvidas do que aqueles com coloproctite. Entretanto, Veloso et al ${ }^{(24)}$ não identificaram correlação entre a presença de artrite e o segmento do cólon acometido nos 343 indivíduos com RCU estudados.

A extensão, localização, duração e o aparecimento de complicações da DII como as fístulas e estenoses, bem como a ressecção do local inflamado, não alteram a evolução da EA ou da sacroiliíte nos pacientes com DC ou com RCU ${ }^{(4,44)}$.

\section{HLA B27}

Datam de 1973 os primeiros relatos da associação do HLA B27 com a EA, logo seguidos pela descrição dessa associação também com a artrite reativa, com a artrite psoriásica, com a EA associada à DC e à RCU, com a espondilite juvenil e com a uveíte anterior aguda ${ }^{(7,8,9)}$. A associação entre o HLA B27 e as espondiloartropatias permanece um dos melhores exemplos da correlação entre uma doença e um marcador genético ${ }^{(58)}$. O HLA B27 está fortemente associado com a susceptibilidade às doenças do grupo das espondiloartropatias, porém a intensidade da associação varia de acordo com a enfermidade, e com os grupos raciais e étnicos ${ }^{(24)}$. Assim, o antígeno HLA B27 ocorre em mais de 90\% dos pacientes com EA, em 60 a 70\% dos indivíduos com artrite reativa, em 40 a $70 \%$ dos doentes com uveíte anterior não granulomatosa, em 35 a 75\% dos pacientes com DC e RCU, e em 30 a 40\% daqueles com sacroiliíte associada à psoríase ou à espondiloartropatia indiferenciada ${ }^{(59)}$. Braun e Sieper ${ }^{(15)}$, em 1996, afirmaram que a associação com o HLA B27 é mais forte nos pacientes com EA e sacroiliíte bilateral, e é claramente mais fraca naqueles com espondiloartropatia e sacroiliíte unilateral.

A prevalência de HLA B27 na população em geral, entretanto, mostra consideráveis variações geográficas, ocorrendo desde $50 \%$ em indígenas ao norte do Canadá ${ }^{(60)}$, a virtualmente ausente em negros africanos ${ }^{(61)}$. As publicações de autores brasileiros indicam uma freqüência do HLA B27 na população brasileira de $3,5 \%^{(62)}$ e $4 \%^{(63)}$. Encontrou-se positividade para o HLA B27 nas espondiloartropatias variando de $46,1 \%$ a $60,9 \%{ }^{(64-66)}$ e na $\mathrm{EA}$, variando de $64 \%$ a $90 \%{ }^{(66-70)}$. Assim, a prevalência de HLA B27 numa população pode apresentar um impacto significativo na ocorrência de enfermidades relacionadas ao antígeno. Deve-se, portanto, interpretar as taxas relatadas de prevalência das diversas doenças associadas ao HLA B27 considerando-se a freqüência deste antígeno na população estudada.

Estudos de mapeamento genético têm implicado outros genes, que não o HLA B27, tanto aqueles que fazem parte do sistema HLA, quanto outros que não pertencem a esse sistema, na susceptibilidade às espondiloartropatias ${ }^{(71)}$. Após investigar 70 famílias francesas de pessoas com espondiloartropatias - o paciente e seus parentes de primeiro grau - e comparar com a 
população francesa normal, Said-Nahal et al ${ }^{72)}$ identificaram que o HLA DR4 tem influência na predisposição genética para este grupo de doenças, tanto para a forma periférica quanto para a axial. Em estudo anterior, Miehle et $a^{(73)}$, haviam afirmado a susceptibilidade maior para a artrite periférica, associada a este gene. A freqüência aumentada de lesões inflamatórias no intestino de pacientes com espondiloartropatias e a associação já conhecida com a DC e a RCU, favorecem a hipótese de um elo patogênico entre intestino e as espondiloartropatias, diferente do HLA B27 ${ }^{(16)}$.

Vários autores têm demonstrado que na artropatia associada à DC e à RCU, o envolvimento axial acha-se associado ao HLA B27, diferente da artrite periférica ${ }^{(26,31,74,77)}$. Scarpa et al ${ }^{(6)}$ identificaram maior freqüência de HLA B27 nos pacientes com RCU ativa que apresentaram EA, quando comparados com o grupo com oligoartrite periférica, e com o grupo sem acometimento articular. Segundo revisão de Reveille ${ }^{(58)}$, de 50 a $60 \%$ dos indivíduos com espondilite, em associação com a DC e a RCU, possuem o gene para HLA B27. Purrmann et al ${ }^{56)}$ identificaram o gene em $72 \%$ dos pacientes com DC e, EA segundo os critérios de Nova York, e em apenas 5\% daqueles com DC e sem espondilite.

A freqüência do HLA B27 na EA associada à DC e à RCU é menor do que na EA idiopática ${ }^{(74,75,76)}$. Autores que analisaram a freqüência do HLA B27 em séries de pacientes com DC e RCU encontraram resultados diversos, que variaram de $3,9 \%$ a $60 \%(6,22,25,28,29,31,75,77)$. Em estudo desenvolvido no Brasil, este antígeno estava presente em 5,6\% dos pacientes com DC e RCU, segundo descrito por Lanna ${ }^{(36)}$.

A freqüência das manifestações articulares e do HLA B27 em pacientes com DC e RCU descrita em alguns estudos publicados após a definição dos critérios de classificação das espondiloartropatias, e dos trabalhos brasileiros, está demonstrada na Tabela 1.

\section{CONSIDERAÇÕES FINAIS}

A identificação da presença de manifestações articulares em pacientes com DC e RCU não significou, simplesmente, a necessidade do reconhecimento destas manifestações clínicas. Trouxe, também, no campo da Reumatologia, novos dados que têm auxiliado no esclarecimento dos fatores etiopatogênicos de enfermidades reumáticas específicas, classificadas dentro do conceito de espondiloartropatias. Tais conhecimentos melhoraram a compreensão da história natural das doenças pertencentes ao grupo e facilitaram a monitorização dos pacientes, permitindo propostas terapêuticas mais apropriadas ${ }^{(17,34)}$.

TABELA 1

FREQÜÊNCIA DAS ALTERAÇÕES ARTICULARES E DO HLA B27 EM SÉRIES DE PACIENTES COM DOENÇA DE CROHN E RETOCOLITE ULCERATIVA, SEGUNDO VÁRIOS AUTORES

\begin{tabular}{|c|c|c|c|c|c|c|}
\hline Autor (ano) & $\begin{array}{c}\text { Doença Crohn } \\
\mathrm{n}\end{array}$ & $\begin{array}{c}\text { Retocolite Ulcerativa } \\
\mathrm{n}\end{array}$ & $\begin{array}{l}\text { Artrite } \\
\mathrm{n}(\%)\end{array}$ & $\begin{array}{c}\text { Sacroiliíte } \\
\mathrm{n}(\%)\end{array}$ & $\begin{array}{c}\text { Espondilite Anquilosante* } \\
\text { n (\%) }\end{array}$ & $\begin{array}{c}\text { HLA B27 + } \\
(\%)\end{array}$ \\
\hline Goldfarb et al (1985) $)^{\mathrm{a}, \mathrm{c}}$ & - & 98 & $14(4,3)$ & - & - & - \\
\hline Scarpa et al (1992) & - & 79 & $23(29,1)$ & $34(43)$ & $20(25,3)$ & 12,2 \\
\hline Maeda et al (1994) b & 203 & - & $21(10,3)$ & - & $3(15)$ & - \\
\hline Veloso et al (1996) & 449 & 343 & $128(16,2)$ & - & $23(2,9)$ & - \\
\hline Silva et al (1998) & 35 & 27 & $19(30)$ & $15^{*}(24)^{* *}$ & $2(3,7)$ & 24 \\
\hline Orchard et al (1998) ${ }^{\mathrm{b}}$ & 483 & 976 & $108(7,4)$ & - & $15(1)$ & - \\
\hline Triantafillidis et al (2000) & 155 & - & $46(30)$ & - & - & - \\
\hline De Vlam et al (2000) & 78 & 25 & $10(10)$ & $33(32)$ & $10(10)$ & 9,2 \\
\hline Salvarani et al (2001) ${ }^{b}$ & 59 & 98 & $17(10,6)$ & $5(3,6)$ & $5(3,1)$ & 3,6 \\
\hline Palm et al $(2001)^{b}$ & 133 & 273 & $18(3,5)$ & - & - & - \\
\hline Palm et al $(2002)^{b}$ & 133 & 273 & - & $8(2)$ & $15(3,7)$ & 13 \\
\hline Christodoulou et al (2002) c & 37 & 215 & $7(2,8)$ & $15(6)$ & - & - \\
\hline Souza et al (2002) $)^{a, c}$ & 126 & 131 & $37(14,4)$ & - & - & - \\
\hline Al-Shamali et al (2003) ${ }^{c}$ & - & 90 & $8(8,9)$ & - & - & - \\
\hline Lanna (2004) a & 71 & 59 & $33(25,4)$ & $12(9,2)$ & $8(6,2)$ & 5,6 \\
\hline
\end{tabular}

* - preencheram critérios de classificação; ${ }^{* *}$ - assintomáticos; ${ }^{a}$ - estudo brasileiro; ${ }^{\text {b }}$ - estudo multicêntrico; ${ }^{c}$ - estudo retrospectivo 


\section{REFERÊNCIAS}

1. Damião AOMC, Sipahi AM: Doença Inflamatória Intestinal. In: Gastroenterologia. Rio de Janeiro: MEDSI Editora Médica e Científica Ltda p.1105-1149, 2004.

2. Danzi JT: Extraintestinal manifestions of idiopathic inflammatory bowel disease. Arch Intern Med 148: 297-302, 1988.

3. Ferrari MLA, Cunha AS: Doenças inflamatórias intestinais. In: Pedroso ERP, Rocha MOC, Silva OA. Clínica Médica - Os Princípios da Prática Ambulatorial. (Ed.) Rio de Janeiro: Livraria Atheneu Editora cap. 71, p. 941- 976, 1993

4. Gravallese EM, Kantrowitz FG: Arthritic manifestations of inflammatory bowel disease. Am J Gastroenterol 83: 703-709, 1988.

5. Wright V, Watkinson G: The arthritis of ulcerative colitis. Medicine 55: 243-259, 1959

6. Scarpa R, Del Puente A, D'Arienzo A, Girolamo C, Valle G, Panarese A, Lubrano E, Oriente P. The arthritis of ulcerative colitis: clinical and genetics aspects. J Rheumatol 19: 373-376, 1992.

7. Brewerton DA, Hart F, Nicholls A, Caffrey M, James DCO, Sturrock RD: Ankylosing spondylitis and HLA B27. Lancet 1: 904-907, 1973.

8. Schlosstein L, Terasaki TI, Bluestone RT: High association of an HL-A antigen, W 27, with ankylosing spondylitis. N Engl J Med 288: 704-706, 1973

9. Brewerton DA. HLA B27 and the inheritance of susceptibility to rheumatic disease. Arthritis Rheum 19: 656-668, 1976.

10. Mielants H, Veys EM, Cuvelier C et al: The evolution of spondyloarthropathies in relation to gut histology. III Relation between gut and joint. J Rheumatol 22: 2279-2284, 1995.

11. Braun J, Bollow M, Remlinger G et al: Prevalence of spondyloarthropathies in HLA B27 positive and negative blood donors. Arthritis Rheum 41: 58-67, 1998.

12. Stafford L, Youssef PP: Spondyloarthropathies: an overview. Int Med J 32: 40-46, 2002

13. Moll JMH, Haslock I, Macrae IF, Wright V: Association between ankylosing spondylitis, psoriatic arthritis, Reiter's disease, the intestinal arthropathies, and Behcet's syndrome. Medicine 53 : 343-463, 1974

14. Mielants H, De Vos M, Cuvelier C, Veys EM: The role of gut inflammation in the pathogenesis of spondiloarthropathies. Acta Clinica Belgica 51: 340-349, 1996

15. Braun J, Sieper J: The sacroiliac joint in the spondyloarthropaties. Curr Op Rheumatol 8: 275-287, 1996.

16. Khan MA: Update on Spondyloarthropathies. Ann Intern Med 136: 896-907, 2002.

17. Dougados M, Hochberg MC. Why is the concept of spondyloarthropathies important? Best Pract Res Clin Rheumatol 16: 495-505, 2002.

18. Wright V, Watkinson G: The arthritis of ulcerative colitis. Brit Med J 2: 670-675, 1965

19. Wright V, Watkinson G: Sacro-iliitis and ulcerative colitis. Brit Med J 2:675-680, 1965

20. Haslock I, Wright V: The muskulo-esqueletal complications of Crohn's disease. Medicine 52: 217-225, 1973

21. Dekker-Saeys BJ, Meuwiessen SGM, Van der Bergen-Loonen EM, De Haas WHD, Agenant D, Tytgat GNJ: Prevalence of peripheral arthritis, sacroiliitis and ankylosing spondylitis in patients suffering from inflammatory bowel disease. Ann Rheum
Dis 37: 33-35, 1978.

22. Münch H, Purrmann J, Reis HE et al: Clinical features of inflammatory joint and spine manifestations in Crohn's Disease. Hepato-gastroenterol 33: 123-127, 1986.

23. Maeda K, Okada M, Yao T, et al: Intestinal and extraintestinal complications of Crohn's disease: predictors and cumulative probability of complications. J Gastroenterol 29: 577-582, 1994.

24. Veloso FT, Carvalho J, Magro F: Immune-related systemic manifestations of inflammatory bowel disease. A prospective study of 792 patients. J Clin Gastroenterol 23: 29-34, 1996.

25. Silva RQ, Garcia JB, Sanchez AF, et al: Artropatia axial silente en la enfermedad inflamatoria intestinal. Caracteristicas clinicas, radiológicas y genéticas. Rev Clin Espanhola 198: 124-128, 1998.

26. Orchard TR, Wordsworth BP, Jewell DP: Peripheral arthropathies in inflammatory bowel disease: their articular distribution and natura history. Gut 42: 387-391, 1998.

27. Triantafillidis JK, Emmanouilidis A, Manousos O, Nicilakis D, Kogevinas M: Clinical Patterns of Crohn`s Disease in Greece: a follow-up study of 155 cases. Digestion 61: 121-128, 2000.

28. Salvarani C, Vlachonikolis IG, Van der Heidje DM et al: Musculoskeletal manifestations in a population-based cohort of inflammatory bowel disease patients. Scand J Gastroenterol 12: 1307-1313, 2001

29. De Vlam K, Mielants H, Cuvelier C, De Keyser F, Veys EM, De Vos M: Spondyloarthropathy is underestimated in inflammatory bowel disease: prevalence and HLA association. J Rheumatol 27: 2860-2865, 2000.

30. Palm O, Moum B, Ongre A, Gran JT: The prevalence and incidence of peripheral arthritis in patients of inflammatory bowel disease, a prospective population-based study (the IBSEN Study). Rheumatol 40: 1256-1261, 2001.

31. Palm O, Moum B, Ongre A, Gran JT: Prevalence of Ankylosing spondylitis and other spondyloarthropathies among patients with inflammatory bowel disease: a population study (The IBSEN Study). J Rheumatol 29: 511-515, 2002.

32. Christodoulou DK, Katsanos KH, Kitsanou M, Stergiopoulou C, Hatzis J, Tsianos EV: Frequency of extraintestinal manifestations in patients with inflammatory bowel disease in Northwest Greece and review of the literature. Dig Liver Dis 34: 781-786, 2002.

33. Dougados M, Van der Linden SR, Huitfeldt B, et al: The European Spondiloarthropathy Study Group: The European Spondiloarthropathy Study Group preliminary criteria for the classification of spondiloarthropathy. Arthritis Rheum 34: 12181227,1991 .

34. Goldfarb M, Leite N, Rubistein J et al: Manifestações reumáticas na retocolite ulcerativa idiopática. Estudo de 98 casos no HSE e revisão da literatura. Rev Bras Reumatol 25: 53-56, 1985.

35. Souza MHLP, Troncon LEA, Rodríguez CM et al: Evolução da ocorrência (1980-1999) da doença de Crohn e da retocolite ulcerativa idiopática e análise de suas características clínicas em um Hospital Universitário do sudeste do Brasil. Arq Bras Gastroenterol 339: 98-105, 2002.

36. Lanna CCD: Manifestações articulares, cutâneas, oculares, e pesquisa do HLA B27 em pacientes com Doença de Crohn e Retocolite Ulcerativa atendidos em Hospital de referência em Minas Gerais 147f. (Tese Doutorado em Medicina) - Faculdade de Medicina, Universidade Federal de Minas Gerais. 2004.

37. Mielants H, Veys EM: The gut in the spondyloarthropathies. J Rheumatol 17: 7-10, 1990.

38. Mielants H, Veys EM, Goemaere S, Goethals K, Cuvelier C, De Vos 
M: Gut inflammation and spondyloarthropathies: clinical, radiologic, biologic and genetic features in relation to the type of histology. A prospective study. J Rheumatol 18: 1542-1551, 1991.

39. Mielants H, Veys EM, Cuvelier C et al: The evolution of spondyloarthropathies in relation to gut histology. II. Histological aspects. J Rheumatol 22: 2273-2278, 1995.

40. De Vos M, Mielants H, Cuvelier C, Elewaut A, Veys E: Long-term evolution of gut inflammation in patients with spondyloarthropathy. Gastroenterol 110: 1696-1703, 1996.

41. De Keyser F, Mielants H: The gut in ankylosing spondylitis and other spondyloarthropathies: inflammation beneath the surface. J Rheumatol 30:2306-2307, 2003.

42. Neumann V, Wright V: Artrhitis associated with bowel disease. Clin Gastroenterol 1983; 12: 767-793.

43. Smale S, Ravinder SN, Orchard TR, Russell AS, Bjarnason I: Inflammatory bowel disease and spondyloartrhopathy. Arthritis Rheum 44: 2728-2736, 2001.

44. Holden W, Orchard T, Wordsworth P: Enteropathic arthritis. Rheum Dis Clin N Am 29: 513-530, 2003.

45. Al-Shamali MA, Kalaoui M, Patty I, Hasan F, Khajah A, Al- Nakib B: Ulcerative colitis in Kuwait: a review of 90 cases. Digestion 67:218-224, 2003

46. Soren A. Joint affections in regional ileitis. Arch Intern Med 117:78-83, 1966.

47. Lindstrom C, Wramsby H, Ostberg G: Granulomatous arthritis in Crohn`s disease. Gut 13: 257-259, 1972.

48. Hermans PJ, Fievez ML, Descamps CL, Aupaix MA: Granulomatous synovitis and Crohn`s disease. J Rheumatol 11: 710-712, 1984.

49. Mielants H, Veys EM, Goethals K, Van der SC, Ackerman C: Destructive lesions of small joints in seronegative spondyloarthropathies: relation to gut inflammation. Clin Exp Rheumatol 8: 23-7,1990.

50. Maher JM, Strosberg JM, Rowley RF, Farber M: Jaccoud's arthropathy and inflammatory bowel disease. J Rheumatol 19: 1637-1639, 1992.

51. Norton KI, Eichenfield AH, Rosh JR, Stern MT, Hermann G: Atypical arthropathy associated with Crohn's disease. Am J Gastroenterol 88: 948-952, 1993.

52. Boyer F, Fontanges E, Miossec P: Rheumatoid arthritis associated with ulcerative colitis: a case with severe flare of both diseases after delivery. Ann Rheum Dis 60: 901, 2001.

53. Ball GV: Arthritis and Inflammatory Bowel Disease. Alabama J Med Scienc 22: 35-36, 1985.

54. Ansell BM, Wigley RAD: Arthritic manifestations in regional enteritis. Ann Rheum Dis 23: 64-72, 1964.

55. Hyla JF, Franck WA, Davis JS: Lack of association of HLA-B27 with radiographic sacrroiliitis in inflammatory bowel disease. J Rheumatol 3: 196-200, 1976.

56. Purrmann J, Zeidler H, Bertrams J et al: HLA antigens in ankylosing spondylitis associated Crohn`s disease. Increased frequency of the HLA phenotype B27, B44. J Rheumatol 15: 1658-1661, 1988.

57. Greenstein AJ, Janowitz HD, Sachar DB: The extraintestinal complicaytions of Crohn's disease and ulcerative colitis: a study of 700 patients. Medicine 55: 401-412, 1976.

58. Reveille JD: HLA B27 and the seronegative spondyloarthropathies. Am J Med Scienc 316: 239-249, 1998.

59. Donadi E: Aspectos moleculares do complexo principal de histocompatibilidade: como entender a associação entre o sistema HLA e as doenças reumáticas. Rev Bras Reumatol 41:
225-236, 2001.

60. Gofton JP: Epidemiology, tissue type antigens and Bechterew`s syndrome (ankylosing spondylitis) in various ethnical populations. Scand J Rheumatol 32: 166-168, 1980.

61. Khan MA: HLA and ankylosing spondylitis. In: Calabro JJ, Carson DW. Rheumatology England, MTP p.23-44, 1987.

62. Moraes Jr. HV: Uveíte anterior aguda e HLA B27: valor diagnóstico e prognóstico. Rev Bras Oftalmol 55: 53-62, 1996.

63. Ferreira E, Ward FE, Amos DB: HL-A in a Brasilian population: evidence for new HL-A specificities-histocompatibility testing 1975. In: Kissmeyer-Nielsen G. Copenhagen, Munksgaard, 1975.

64. Carvalho MAP, Campos WR, Araujo CAA, Lacerda RR, Orefice F: Uveítes anteriores não granulomatosas, espondiloartropatias e HLA B27. Rev Bras Reumatol 39: 195-202, 1999.

65. Faustino PC, Terreri MT, Andrade CT, Len C, Hilário MO: Características clínicas das espondiloartropatias na infância: análise de 26 pacientes. Rev Ass Med Bras 47: 216-220, 2001.

66. Bomtempo CAS: Avaliação clínica, laboratorial e radiográfica de pacientes com espondiloartropatias, com especial referência ao antígeno leucocitário humano B27. 123f. Tese (Mestrado em Medicina) - Faculdade de Medicina, Universidade Federal de Minas Gerais, Belo Horizonte, 2004.

67. Chahade WB, El-Koury AB, Szwarc IS: Incidência do HLA-B27 em espondilite anquilosante em caucasóides brasileiros. Associação dos locos A, B e C/ espondilite anquilosante/ tuberculose pulmonar. Rev Bras Reumatol 19: 23-26, 1979.

68. Rachid A: Espondilite anquilosante B27 positivas e B27 negativas. Cortejo clínico, laboratorial e radiográfico. Rev Bras Reumatol 19: 107-127, 1979.

69. Ramalho ES, Freitas GG, Kosminsky S, Cavalcanti FS, Menezes FC, Leite MSS: Espondilite anquilosante: estudo epidemiológico em trinta casos. Rev Bras Reumatol 29:1-2, 1989.

70. Sampaio-Barros PD, Bertolo MB, Kraemer MH, Marques-Neto JF, Samara AM. Primary Ankylosing spondylitis: Patterns of disease in a brazilian population of 147 patients. J Rheumatol 28: 560-565, 2001.

71. Brown MA, Crane AM, Wordsworth BP: Genetic aspects of susceptibility, severity, and clinical expression in ankylosing spondilitis. Curr Op Rheumatol 14: 354-360, 2002.

72. Said-Nahal R, Miceli-Richard C, Gautreau C et al: The role of HLA genes in familial spondyloarthropathy: a comprehensive study of 70 multiplex families. Ann Rheum Dis 61: 201-206, 2002.

73. Miehle W, Schattenkirchner M, Albert D, Bunge M: HLA DR4 in ankylosing spondylitis with different patterns of joint involvement. Ann Rheum Dis 44: 39-44, 1985.

74. Morris RI, Metzger AL, Bluestone R, Terasaki PI: HL- A- W27 - a useful discriminator in the arthropathies of inflammatory bowel disease. N Engl J Med 290: 1117-1119, 1974.

75. Dekker-Saeys BJ, Meuwiessen SGM, Van der Bergen-Loonen EM, De Haas WHD, Meijers KAFR, Tytgat GNJ: Clinical characteristics and results of histocompatibility typing (HLA B27) in 50 patients with both ankylosing spondilytis and inflammatory bowel disease. Ann Rheum Dis 37: 36-41, 1978.

76. Mielants H, Veys EM, Cuvelier C, De Vos M, Botelberghe L: HLA B27 related arthritis and bowel inflammation. Par 2 . ileocolonoscopy and bowel histology in patients with HLA B27 arthritis. J Rheumatol 12: 294-298,1985.

77. Nagant De Deuxchaisnes C, Huaux JP, Fiasse ER: Ankylosing spondylitis, sacroiliitis, regional enteritis and HLA - A27. Lancet 1:1238,1974. 